\title{
¿Leer para estar bien?: prácticas actuales y perspectivas sobre la biblioterapia como estrategia educativo-terapéutica
}

\author{
Anaclara Castro Santana* \\ Nelly Altamirano Bustamante*
}

Artículo recibido:

1 de marzo de 2016

Artículo aceptado:

27 de septiembre de 2016

\section{Resumen}

La biblioterapia, una tendencia con raíces ancestrales, ha cobrado un nuevo auge mundial en tiempos recientes. Esta propuesta de intervención educativo-terapéutica plantea que es posible mejorar la salud por medio del contacto habitual con la lectura. Partiendo del entendido de que los actos significativos de lectura propician cambios en los individuos, los teóricos y practicantes de la biblioterapia argumentan que leer puede aumentar el bienestar de las personas en diversos contextos y etapas de sus vidas, en especial cuando se hace de manera guiada y propositiva. En este artículo se presenta una revisión de los conceptos y facetas

* Instituto Nacional de Pediatría, México anaclarius@gmail.com acastrosa@conacyt.mx nellyab34@gmail.com

INVESTIGACIÓN BIBLIOTECOLÓGICA, vol.32, núm.74, enero/marzo, 2018, México, ISSN: 2448-8321 pp. 171-192 
actuales de esta práctica interdisciplinaria, seguida de una exploración sobre sus posibilidades de estudio y aplicación a futuro.

Palabras clave: Biblioterapia; Lectura; Salud; Intervención Educativa

Reading oneself to health?: current practices and prospects for bibliotherapy as a didactic-therapeutic tool Anaclara Castro-Santana, Nelly Altamirano-Bustamante

\section{Abstract}

Biblio-therapy, an age-old technique, has recently regained some momentum in diverse settings around the world. This therapeutic-didactic approach posits that one can regain or improve health by reading on a regular basis. Taking this as a point of departure, the idea that significant acts of reading elicit changes in the reader, theorists and active practitioners of bibliotherapy argue that books can improve the well-being of individuals, particularly when it is guided in some way. This paper offers an assessment of concepts and current trends in biblio-therapy as an interdisciplinary practice, along with an exploration of potential paths for further development.

Keywords: Biblio-therapy; Reading; Health; Educational intervention

Come, and take choice of all my library, And so beguile thy sorrow. William Shakespeare, Titus Andronicus (Act IV, Sc1.34)

The liveliness of literature lies in its exceptionality, in being the individual, idiosyncratic vision of one human being, in which, to our delight and great surprise, we may find our own image reflected. A book is a version of the world. If you do not like it, ignore it or offer your own version in return. Salman Rushdie, In Good Faith 


\section{INTRODUCCIÓN}

— $\mathrm{n}$ nuestros días se habla mucho de las bondades de la lectura. Se dice Eque leer es deseable, loable, necesario; que le da sustancia a nuestra fachada. Se trata de un fenómeno mundial, del que México no se encuentra exento. Existen programas gubernamentales e independientes, ferias del libro, talleres y campañas. El fomento a la lectura se ha convertido incluso en epítome de éxito en mercadotecnia, como lo demuestran las ingeniosas frases en fondo amarillo de la cadena de librerías más grande de nuestro país, que recurre a temas de actualidad para vender (de forma simbólica y literal) la idea de que leer genera cambios positivos (Chávez, 2008). Por desgracia, los indicadores revelan que, a pesar de los esfuerzos, México dista de convertirse en un país de lectores (Conaculta 2006 y 2012; Villamil, 2013; Inegi, 2014). Lo que es más, plantear la lectura como obligación intelectual e incluso moral puede propiciar tanto una sobreponderación de sus beneficios como la satanización de quienes no leen, ambas perspectivas potencialmente generadoras de un efecto paradójico de desaliento. En las grandes metrópolis, dicho sea de paso, la lectura con fines recreativos compite por la atención y exiguo tiempo de sus habitantes, a quienes se les presentan alternativas de entretenimiento casero engañosamente más sencillas, como la televisión, los videojuegos y, cada vez más, los contenidos heterogéneos, y a menudo fragmentarios, de internet.

Existen, sin embargo, verdades innegables detrás de las (casi siempre bienintencionadas) campañas del fomento a la lectura, puesto que, efectivamente, leer cambia la manera en que vemos el mundo, enriquece el bagaje cultural y amplía los horizontes de quienes lo hacen de manera habitual; aumenta la capacidad de análisis, ayuda a incrementar el lapso de concentración y forja un carácter más crítico de la realidad. Quienes practican la lectura como actividad cotidiana reconocen su poder: disfrutan de sumergirse en el mundo descrito en las páginas de un buen libro, o de adentrarse en la mente de un personaje bien construido; sienten tristeza, alegría o rabia ante las situaciones descritas y el sufrimiento de personas ficticias; se involucran en sus vidas inexistentes. Al final del día, al reconocer sus propios procesos mentales y los de otros, logran tener mayor entendimiento sobre la condición humana. Quizá lo que hace falta, entonces, es acercar los libros de manera más activa y sin señalamientos acusatorios contra quienes se sienten ajenos al mundo de las letras.

Por otra parte, haciendo una breve digresión, los adelantos científico-tecnológicos que han permitido lograr avances significativos en la preservación y el restablecimiento físico de la salud han llevado a un olvido 
relativo del carácter multidimensional tanto de los pacientes como del personal médico, de suerte que la atención sanitaria tiende a centrarse únicamente en la enfermedad identificada. Esto lleva a una deshumanización de la práctica clínica que con frecuencia genera insatisfacción en quienes van al médico, aun cuando los aspectos orgánicos de su padecimiento son, en buena medida, atendidos y remediados (Fulford, 2011). Asimismo, existen enfermedades en las que los componentes emocionales y motivacionales resultan determinantes para mejorar la reacción somática. Un ejemplo muy claro lo constituyen los padecimientos de tipo crónico-degenerativo en personas de todas las edades, pero en especial en niños y adolescentes, quienes, por lo general, dependen más de otros y requieren más estímulos exteriores para desarrollar su motivación (Palladino y Helgeson, 2012).

Tomando estos contextos como punto de partida, en las siguientes páginas abordaremos aspectos teóricos y prácticos de la denominada "biblioterapia”, una práctica pedagógico-terapéutica que consiste en un acercamiento guiado a la lectura, en especial en momentos de crisis, con fines de rehabilitación mental y espiritual. Esta técnica, probablemente tan antigua como la escritura misma, plantea que el lenguaje y la narrativa propician cambios determinantes en las personas, lo cual puede llevar a una mejora en su salud, aun cuando no se encuentren enfermos de manera tangible. ${ }^{1}$ Partiendo de la hipótesis de que cuando se entabla una relación significativa con un texto "algo" ocurre dentro de nosotros, la biblioterapia se ocupa de tratar de comprender y utilizar este proceso para aumentar el bienestar de individuos en diversos contextos (instituciones de salud, escuelas, centros comunitarios, centros de rehabilitación social, entre otros) por medio de la lectura dirigida. En este artículo se presenta una revisión de los conceptos y facetas actuales de esta práctica interdisciplinaria, seguida de un análisis sobre sus posibilidades de estudio y aplicación a futuro en diversas áreas.

\section{ObJetivo y mÉtodos de tRABajo}

El presente trabajo tiene como objetivo general explorar el estado de la cuestión y potenciales vías de desarrollo de un concepto importante, que conjunta metodologías de áreas complementarias del conocimiento: educación, bibliotecología, literatura y ciencias de la salud. En las páginas siguientes presentamos una revisión narrativa de la bibliografía existente sobre biblioterapia con

1 En este artículo entenderemos por salud la definición clásica de la OMS (1948: 100): “un estado de completo bienestar físico, mental y social, y no solamente la ausencia de afecciones o enfermedades". 
miras a vislumbrar nuevas formas en que ésta podría estudiarse e implementarse en México de manera más consistente que hasta ahora.

La información que a continuación se detalla proviene de una búsqueda sistemática en bases de datos tanto de ciencias de la salud (MEDLINE/PubMed, Cochrane Library), como de ciencias sociales, humanidades y multidisciplinarias (ERIC, Jstor, Project MUSE, Intute, Worldcat). Los algoritmos de búsqueda (en inglés) utilizados para la obtención de fuentes primarias fueron bibliotherapy, en combinación con bealth hospital, education, bealing, fiction, poetry, literature, library, chronic diseases y self-help, utilizando como delimitador booleano AND y limitando los campos de búsqueda a abstracts, títulos y palabras clave. Se replicó la búsqueda con los términos en español y sus combinaciones: "biblioterapia", "hospital", "salud", "sanar", "narrativa", "poesía", "literatura", "biblioteca", "enfermedades crónicas", “desarrollo personal". Después de una primera lectura crítica de títulos y resúmenes se realizó una nueva búsqueda agregando el término clave literatherapy/“literapia”. De los resultados arrojados se excluyeron textos anteriores a 1980, con excepción de los artículos de Samuel McChord Crothers y Catherine Shrodes, por ser pioneros en el campo. Por otra parte, se seleccionaron para análisis particular artículos posteriores a 2005 para privilegiar un estudio del estado de la cuestión en la última década. Se realizó también una búsqueda en bases de datos de tesis (TESIUNAM, Proquest Dissertation Abstracts, EThOS) utilizando los criterios y filtros antes mencionados. Para el resumen de los resultados que se detalla a continuación se trató de mostrar de manera implícita la proporción de información encontrada por región y por tipo de intervención. Es decir, puesto que una gran mayoría de los artículos encontrados son de origen anglosajón, nuestro reporte se muestra inclinado hacia esa región; asimismo, puesto que es de nuestro interés buscar formas de explorar e implementar la biblioterapia en México, se dio atención particular a textos latinoamericanos en una proporción que no es imparcialmente representativa de la literatura existente en el mundo. Al analizar de manera crítica las publicaciones seleccionadas surgieron temas comunes a la mayoría, que se detallan en las secciones que componen este artículo, a saber: definiciones del concepto, historia y práctica de la biblioterapia, tipos de biblioterapia (creativa o clínica, de acuerdo con la clase de textos utilizada), ámbitos de implementación y perfil del biblioterapeuta. Cabe mencionar que la revisión puso de manifiesto una notoria heterogeneidad en cuanto al tipo de evaluaciones e instrumentos utilizados para valorar los efectos de la terapia. 
Existe una gama amplia de definiciones de biblioterapia que van desde propuestas escuetas como "leer para tratar enfermedades" (Dysart-Gale, 2008: 33), "ayudar con libros" (Jones, 2006: 24), hasta ejemplos más complejos como el de Stephen Bonnycastle, quien postula que

la biblioterapia se enfoca en un proceso central que ocurre en los actos de lectura más significativos: un libro entra en la vida de un individuo; se establece una relación profunda y la persona cambia como consecuencia de este involucramiento. La biblioterapia aborda cómo y porqué ocurre esto, así como las maneras en que dicho proceso puede ser dirigido de forma que nos ayude a mejorar nuestra vida desde lo individual y como miembros de una sociedad. (Bonnycastle, 2001: 1. Traducción propia)

Una de las definiciones más aceptadas es la del Diccionario de Bibliotecología y Ciencias de la Información, en donde se describe a la biblioterapia como "el uso de libros, seleccionados con base en su contenido, leídos como parte de un programa diseñado para facilitar la recuperación de pacientes que sufren alguna enfermedad mental o un malestar emocional como consecuencia de su enfermedad" (Reitz, 2014: 5). Algunos difieren en cuanto a si la biblioterapia puede considerarse como tal cuando se da con participantes sanos, si debe hacerse con textos de autoayuda o de literatura creativa, si es mejor realizarla en grupo o de manera individual, si se requiere un coordinador que imparta el taller o si sus beneficios son mayores en niños, jóvenes o adultos. Lo que todas estas definiciones tienen en común es ver al texto escrito como vehículo para lograr una transformación en la perspectiva de quien lee con respecto a su realidad o estado mental. De acuerdo con las perspectivas teóricas de los diversos autores estudiados en esta revisión, los beneficios de la lectura guiada estriban en la complementariedad de la activación de procesos cognitivos complejos (de ahí las implicaciones didácticas de la biblioterapia) y el involucramiento emocional con las situaciones leídas.

La Tabla 1 enlista una muestra representativa de definiciones del concepto, desde su introducción en el ámbito académico en la década de 1950 hasta nuestros días.

\begin{tabular}{|l|l|}
\hline $\begin{array}{l}\text { Russell y Shrodes } \\
\text { (1950) }\end{array}$ & $\begin{array}{l}\text { It is a process of dynamic interaction between the personality of the reader and imagi- } \\
\text { native literature-interaction which may be utilized for personality assessment, adjust- } \\
\text { ment, and growth. (355) }\end{array}$ \\
\hline $\begin{array}{l}\text { Jalongo } \\
1983)\end{array}$ & $\begin{array}{l}\text { One strategy for reinforcing a language arts approach to reading by guiding children to } \\
\text { personal and significant experiences with books. (796) }\end{array}$ \\
\hline
\end{tabular}




\begin{tabular}{|l|l|}
\hline $\begin{array}{l}\text { Marrs } \\
\text { (1995) }\end{array}$ & $\begin{array}{l}\text { The use of written materials or computer programs, or the listening/viewing of audio/ } \\
\text { videotapes for the purpose of gaining understanding or solving problems relevant to a } \\
\text { person's developmental or therapeutic needs. The goals of the bibliotherapy should be } \\
\text { relevant to the fields of counselling and clinical psychology. (846) }\end{array}$ \\
\hline $\begin{array}{l}\text { Salaverti Pitarch } \\
\text { (1999) }\end{array}$ & $\begin{array}{l}\text { Técnica auxiliar de la medicina para mejorar el estado psicológico de los enfermos } \\
\text { combatiendo el aburrimiento, la soledad, la melancolía, la ansiedad, la pérdida de in- } \\
\text { timidad. (75) }\end{array}$ \\
\hline $\begin{array}{l}\text { Brewster } \\
(2009)\end{array}$ & $\begin{array}{l}\text { Creative bibliotherapy —-the use of fiction and poetry to work with individuals and } \\
\text { groups to promote better mental health. } \\
\text { Self-help bibliotherapy —-the use of nonfiction self-help books, often recommended by } \\
\text { medical practitioners, to provide practical help to people with mental health problems. } \\
\text { (400) }\end{array}$ \\
\hline $\begin{array}{l}\text { Pérez de Rosell } \\
(2011)\end{array}$ & $\begin{array}{l}\text { La ejecución de programas con actividades de lectura para pacientes hospitalizados, o } \\
\text { recluidos en áreas de salud, hospicios y hasta prisiones. (111) }\end{array}$ \\
\hline $\begin{array}{l}\text { Akinola } \\
(2014)\end{array}$ & $\begin{array}{l}\text { The process of using books to help children think about, understand, and work through } \\
\text { social and emotional concerns. (1281) }\end{array}$ \\
\hline
\end{tabular}

Tabla 1. Definiciones de biblioterapia Fuente: elaboración propia

Es importante destacar que existe un cierto grado de heterogeneidad en cuanto a la terminología que se utiliza para designar a lo que en este documento nos referimos como biblioterapia. Se le ha llamado biblioguía, literapia, terapia de lectura, biblioorientación, bibliopsicología, libro-cotejo, bibliodiagnóstico, biblioprofilaxis, videoterapia, literapéutica, entre otros (Pehrsson, 2005; Cohen, 1994). ${ }^{2}$ Si bien en ocasiones las variaciones ponen énfasis en un aspecto específico (por ejemplo, la bibliopsicología, que se enfoca de manera más puntual en los efectos terapéuticos de la lectura sobre la conducta y los procesos mentales), en muchos casos los cambios de nomenclatura responden a preferencias individuales por parte de los autores.

La formación académica y profesional del biblioterapeuta es, de igual forma, fuente de controversia entre los investigadores y practicantes de la terapia de lectura. El término "terapia", que hace pensar en un contexto exclusivo de las ciencias de la salud (mental), es sin duda una causa importante detrás de este tipo de debate. Mientras que algunos proponen que debe ser un profesional de la salud o un psicoterapeuta capacitado para dar orientación especializada (Kramer, 2009; Turner, 2008; Reeves, 2010), otros sitúan a los bibliotecólogos, pedagogos, maestros de educación básica y profesores de literatura en el centro de la intervención biblioterapéutica (Bernstein, 1989; Gladding, 2005; Manecke, 2009). La mayoría de los investigadores,

2 Los términos arriba mencionados son nuestras traducciones de vocablos en inglés (en su mayoría neologismos): biblioguidance, reading cure, biblio-counselling, bibliopsychology, book-maching, bibliodiagnostics, biblioprophylaxis, videotherapy y literatherapy. 
sin embargo, apuestan por la interdisciplinaridad en el abordaje de la biblioterapia (Anglin, 2008; McMillen, 2006; Pérez de Rosell, 2011; Duffy et al., 2013: 3-5; Schechtman, 2009: 105-110; García y Bregoli, 2000). Tras realizar un análisis de las propuestas que se reportan en el presente texto, salta a la vista que las más exitosas son aquellas en las que se combinan los conocimientos de diversas disciplinas. Consideramos que para lograr despertar el interés y la empatía de los participantes, es indispensable que quien imparte el taller tenga amplio conocimiento literario, así como buena experiencia con manejo de grupo. Asimismo, resulta muy útil contar con la asesoría de un profesional de la salud mental, que pueda alertar sobre posibles riesgos al abordar temas que pueden resultar delicados en grupos etarios o sociales vulnerables (por ejemplo, adolescentes, adultos mayores, personas en situación de duelo, entre otros). A final de cuentas, lo más importante es que el equipo que planea e imparte la biblioterapia conozca a su población meta, de suerte que pueda diseñar un programa a la medida de sus participantes. Si bien algunas organizaciones independientes ofrecen cursillos y programas para certificarse como biblioterapeuta o facilitador de biblio y poeterapia, hasta la fecha no existen licenciaturas o posgrados específicos para la profesionalización del biblioterapeuta, lo cual nuevamente pone de manifiesto la necesidad de combinar conocimientos y experiencias de profesionales de diversas áreas para lograr programas de biblioterapia más exitosos. ${ }^{3}$

\section{Breve historia de la biblioterapia}

Aunque el término es de acuñación relativamente reciente, a lo largo de la historia los seres humanos se han aproximado al arte y la literatura para provocar reacciones emocionales (e incluso somáticas), para mitigar el malestar de enfermedades físicas o mentales, o simplemente atemperar el hastío producto de la vida cotidiana. En la era dorada de la civilización egipcia, por mencionar uno de los casos más conocidos, el faraón Ramsés II diseñó la biblioteca más antigua de que se tiene noticia, ubicada en Tebas, ${ }^{4}$ enmarcando el portal de entrada con la inscripción "la casa para sanar el alma”, según lo reporta el historiador griego Diodoro de Sicilia (60-30 A. de C.) (Diodoro Sículo, 2001: 175). Otro ejemplo paradigmático es el concepto aristotélico de “catarsis", esto es, la "purga" o "limpieza” simbólica que la tragedia dramática debe provocar en los espectadores (Aristóteles, s. f./1948: 69-91).

3 Por ejemplo, aquellos que se ofrecen por International Federation for Biblio/Poetry Therapy y Well into Words, Kirklees.

4 No debe confundirse la ciudad egipcia de Waset, más conocida por su nombre griego Tebas, con la ciudad griega también llamada Tebas, como sucede en algunos artículos consultados. 
La adopción sistematizada del concepto de biblioterapia comienza a darse en los albores del siglo XX, a partir de la Primera Guerra Mundial, cuando enfermeras británicas recurrieron a la lectura de textos clásicos en los pabellones hospitalarios como tratamiento complementario para pacientes que experimentaban síntomas de la llamada "neurosis de guerra" (shell shock) (Rozalski, Stewart y Miller, 2010). En este contexto, uno de los pioneros en ofrecer una definición clara y una propuesta concreta de biblioterapia fue Samuel McChord Crothers, quien en 1916, de manera un tanto juguetona, detalló cómo los libros podían usarse como prescripción médica, argumentando que podían servir como ingrediente básico de curación, adyuvante en el tratamiento correctivo para disminuir los efectos negativos o simplemente como vehículo para hacer el padecimiento más llevadero (McChord Crothers, 1916). La biblioterapia hospitalaria tuvo un nuevo auge al término de la Segunda Guerra Mundial, nuevamente en el tratamiento de soldados y otras personas afectadas física y mentalmente por el conflicto armado. A partir de la década de 1950 este campo empieza a consolidarse como área de estudio académico y programa de terapia grupal, en el que un profesional de la salud mental o un experto en literatura (o alguien que posea conocimiento de ambas áreas) funge como guía para la selección y discusión de materiales bibliográficos de relevancia para todos los miembros. La académica norteamericana Caroline Shrodes se convirtió en una de sus impulsoras más destacadas entre 1950 y 1960, al formular las bases teóricas para la práctica metódica de la biblioterapia, especialmente con literatura creativa (Shrodes, 1950 y 2005). A partir del trabajo de estos pioneros, y gracias a adelantos en los campos de las neurociencias por un lado y al reconocimiento de la importancia de la empatía para el bienestar del individuo por el otro, en décadas recientes se han revalorado las posibilidades de la biblioterapia para generar cambios positivos, tanto emocionales como cognitivos, en las personas. En las páginas siguientes haremos una semblanza de los principales modelos que se han propuesto en los últimos 20 años.

\section{Tipos de biblioterapias}

Como hemos mencionado antes, en la actualidad existen debates con respecto a los usos de la biblioterapia y la manera en que debe(ría) impartirse. Por esta razón, los estudiosos de la materia han recurrido a una taxonomía dicotómica que divide a la biblioterapia en dos tipos primordiales: creativa y clínica. En algunos casos la nomenclatura obedece al contexto en donde se realiza la intervención (centro de salud o ámbito educativo), y en otros casos de los materiales a los que se recurre (textos de autoayuda y desarrollo personal, 
u obras literarias de diversos géneros). La biblioterapia clínica recibe otros nombres como biblioterapia de autoayuda y libros por prescripción, mientras que a la biblioterapia creativa frecuentemente se le conoce como literapia (Hicks, 2006; Kramer, 2009; Brewster, 2009).

\section{Clínica}

La denominada biblioterapia clínica o de autoayuda se practica principalmente en el ámbito de la salud mental, con una aproximación metodológica basada en gran medida en meta-análisis de estudios controlados, encaminados a evaluar la efectividad de recomendar lecturas dirigidas a determinado padecimiento, como, por ejemplo, la depresión (Cujipers, 1997; Gregory et al., 2004; Floyd, 2003), la ansiedad (Dobson, 2003; Sharma et al., 2014), el alcoholismo (Apodaca y Miller, 2003), o los desórdenes alimenticios (Rodríguez-Martín et al., 2013). La gran mayoría de estas investigaciones han llevado a la conclusión de que la biblioterapia con textos especializados en desarrollo personal es efectiva en ciertos casos, a saber: cuando la severidad del padecimiento va de leve a moderada, cuando se combina con otros tratamientos psicológicos (por ejemplo terapia cognitivo-conductual), o cuando las circunstancias espaciotemporales limitan las posibilidades de contacto entre los pacientes y el terapeuta. Este modelo de biblioterapia con frecuencia es diseñado e implementado por grupos multidisciplinarios que combinan el conocimiento y experiencia de médicos, personal de enfermería, psicoterapeutas y bibliotecólogos. En ocasiones, como en el caso del esquema británico Books on Prescription, es el sistema nacional de salud (NHS, por sus siglas en inglés) quien diseña y financia la propuesta, pero los programas se implementan fuera del ámbito clínico, en espacios sociales como bibliotecas públicas y centros comunitarios. Esto último parece funcionar muy bien, dado que al jugar con la idea de que los libros pueden ser una "prescripción" médica, al tiempo que lleva la lectura a espacios más amigables para el público que los contextos hospitalarios, se estimula la curiosidad de las personas. Parecería que el sentido de pertenencia a una comunidad y la formación de vínculos por medio del estudio de un tema de interés común contribuye a potenciar los cambios positivos en las personas que asisten a este tipo de biblioterapia. Por otra parte, la realización de talleres en bibliotecas públicas permite contar con mayor cantidad y diversidad de materiales que logren captar la atención de lectores con intereses muy variados. 


\section{Creativa}

La biblioterapia creativa -a la que de aquí en delante nos referiremos como literapia para facilitar la distinción- ha recibido menos atención desde el punto de vista estrictamente científico. Esto no quiere decir que se practique con menor frecuencia, ni que su aplicación sea menos exitosa o de menor relevancia. Quienes abogan por la práctica de la literapia plantean que al inducir la lectura de obras narrativas de ficción se puede lograr un involucramiento espontáneo entre los participantes (llámense pacientes, internos, alumnos, usuarios de biblioteca, etcétera) y los libros (Shrodes, 1950: 26-28; Brewster, 2011: 70; Deberti Martins, 2011; Akinola, 2014). Este supuesto comparte elementos importantes con ciertas vertientes de teoría literaria que estudian los textos desde el punto de vista de los lectores (la denominada estética de la recepción). De acuerdo con teóricos de esta escuela de interpretación, como Wolfgang Iser, en el acto de lectura se genera una doble referencialidad, o representación: la del mundo narrado y aquello que los lectores agregan a éste en el proceso de interpretación, de suerte que la identidad del lector se amplía y podemos "al mismo tiempo, [ser] nosotros mismos y alguien más" (Iser, 1989: 244). Con la práctica, esto permite a los lectores crear nuevas versiones de sí mismos, más amplias y expresivas. Del mismo modo, se ha sugerido que, en parte gracias al proceso de identificación, la inmersión individual en textos narrativos puede aumentar la confianza en la propia capacidad de actuar, lo cual contrarresta la apatía, que lleva a un pobre control sobre las circunstancias de vida (Louie y Louie, 1992). Como lo plantean algunos críticos, "las formas privadas de lectura [...] refuerzan el auto-empoderamiento del individuo" (Fluck, 2003: 23).

Los teóricos y practicantes de la literapia a menudo comparten estas visiones de manera explícita o implícita. Parten del entendido de que en los relatos narrativos se dan conflictos íntimos e intensos, que al mismo tiempo se presentan alejados del lector (al estar mediados por distancias espacio-temporales), de suerte que la empatía coexiste con la postura crítica de las situaciones narradas. La literatura creativa permite a los lectores identificarse no sólo con el protagonista o con el personaje más obvio, sino con otros de diferente género, edad, posición social o con situaciones de vida ostensiblemente muy distintas.

Los puntos anteriores influyen de manera importante en el marco teórico de la literapia hospitalaria, puesto que se plantea que la identificación con personajes ficticios permite a los pacientes verse a sí mismos y a otros como personas completas y no sólo como enfermos (Gold, 1990: 346-354; Deberti Martins, 2011: 147-149; Pérez de Rosell, 2011: 112-113). De este modo, se propone que a partir de la lectura de obras afines a sus intereses, las personas con 
enfermedades crónicas que requieren cuidado constante y vitalicio pueden lograr una apertura conceptual y un incremento motivacional, que les permita trascender la visión frecuentemente atrapante y catastrófica de su padecimiento desde el punto de vista puramente somático y estadístico (Amer, 1999). Por medio de historias con humor, aventura y suspenso los lectores pueden tener acceso a realidades alternativas que les permiten distraerse de sus preocupaciones, sentirse acompañados y tender puentes de comunicación diversos con sus pares y con sus familiares. Esto es especialmente pertinente para pacientes de enfermedades crónicas, para quienes la motivación y el estado anímico son cruciales. La literapia, sin embargo, no sólo puede resultar terapéutica en personas con una enfermedad manifiesta y reconocible. Como lo plantean las teorías antes mencionadas, cualquier lector puede obtener beneficios de la inmersión cognitiva y emocional que ocurre durante el acto mismo de la lectura. Así pues, podría pensarse que los programas de fomento a la lectura en escuelas y centros comunitarios quizá se beneficiarían de incorporar algunas de las herramientas propuestas por los teóricos y practicantes de la literapia, objetos de estudio en esta revisión. Por ejemplo, en vez de plantear la lectura como un deber, o como una manera de estimular la inteligencia, podría apelarse a los aspectos divertidos y emocionalmente reconfortantes de ésta.

\section{DE LA TEORÍA A LA PRÁCTICA: INTERVENCIONES Y PROGRAMAS en MÉxico y el MUNDo}

En décadas recientes, la biblioterapia, en sus dos vertientes principales, se ha afianzado como un atractivo modelo educativo-terapéutico. Es una estrategia educativa en tanto que la lectura de cualquier tipo proporciona información nueva e instruye; y resulta terapéutica dado que, al mejorar el estado anímico, puede incidir en el bienestar físico y emocional de quienes participan en los talleres de lectura (incluyendo a participantes considerados sanos, pero que, como todas las personas, están expuestos a factores ambientales, sociales y familiares de estrés). Desde al menos la década de 1990, la biblioterapia se ha implementado en diversos países alrededor del mundo, como el Reino Unido (Manecke, 2009), España (Salaverti Pitarch, 1999), Canadá (Tukhareli, 2016), Estados Unidos (McMillen, 2006), Brasil (Seitz, 2008; Bernardino, 2012), Uruguay (Deberti Martins, 2011 y 2013) y Venezuela (Pérez de Rosell, 2011: 112), por mencionar los que aparecen reportados con mayor frecuencia.

Los datos arrojados por nuestra revisión sistemática en bases de datos de publicaciones científicas, sociales y humanísticas, así como de trabajos 
de investigación no publicados (tesis y resúmenes de ponencias), revelan que en México la biblioterapia se realiza de manera sólo esporádica, y en ocasiones los programas tienen una duración muy corta. Por ejemplo, Mosqueda Moreno (2009) reporta un loable proyecto de biblioterapia llevado a las salas de espera y a la biblioteca de un importante hospital oncológico en la Ciudad de México. Sin embargo, cabe destacar que una visita presencial a dicha institución puso de manifiesto que los talleres de lectura se llevaron a cabo durante un periodo relativamente corto (durante la redacción del informe académico que los refiere) y fueron abandonados posteriormente, al no contarse con recursos materiales y humanos para continuar. Un fenómeno similar ocurrió con el programa reportado por Sánchez Trejo (2009), una iniciativa de biblioterapia en un hospital de rehabilitación física. Digno de mención también resulta el hecho de que los programas de biblioterapia que se describen en México con frecuencia se encuentran confinados a la capital del país. Es probable que esto se deba al bien conocido fenómeno de centralización, aunque también es posible que existan prácticas informales de terapia de lectura que, o bien no lleguen a generar reportes indizados, o bien no reciban el nombre de biblioterapia o alguno de sus sinónimos reconocibles. Estos ejemplos comprueban nuestra observación en la sección anterior sobre cómo los programas de fomento a la lectura podrían beneficiarse de integrar los planteamientos de la biblioterapia en sus modelos. De igual forma, los programas de biblioterapia resultarían mucho más exitosos a largo plazo si desde su planteamiento y diseño pudieran acercarse o empatarse con otras iniciativas de fomento a la lectura.

\begin{tabular}{|c|c|c|c|c|c|c|}
\hline Autor/fecha & Lugar & Duración & $\begin{array}{c}\text { Población } \\
\text { (tipo y edad) }\end{array}$ & $\begin{array}{c}\text { Número de } \\
\text { participantes }\end{array}$ & $\begin{array}{c}\text { Perfil del } \\
\text { biblioterapeuta }\end{array}$ & $\begin{array}{c}\text { Tipo de } \\
\text { evaluación/ } \\
\text { indicadores } \\
\text { evaluados }\end{array}$ \\
\hline $\begin{array}{l}\text { Salaverti Pitarch } \\
\text { (1999) }\end{array}$ & $\begin{array}{l}\text { Biblioteca } \\
\text { del Hospital } \\
\text { La Fe, } \\
\text { Valencia, } \\
\text { España }\end{array}$ & $\begin{array}{l}\text { Programa } \\
\text { continuo, } \\
\text { por tiempo } \\
\text { indefinido }\end{array}$ & $\begin{array}{l}\text { Pacientes y } \\
\text { familiares (edad } \\
\text { no especificada) }\end{array}$ & Indefinido & Bibliotecarios & $\begin{array}{l}\text { No se reporta } \\
\text { evaluación }\end{array}$ \\
\hline $\begin{array}{l}\text { McMillen } \\
(2006)\end{array}$ & $\begin{array}{l}\text { Oregon Sta- } \\
\text { te University } \\
\text { (en línea), } \\
\text { Oregon, } \\
\text { EUA }\end{array}$ & $\begin{array}{l}\text { Programa } \\
\text { continuo, } \\
\text { por tiempo } \\
\text { indefinido }\end{array}$ & $\begin{array}{l}\text { Niños y } \\
\text { jóvenes (edad } \\
\text { no especificada) }\end{array}$ & Indefinido & $\begin{array}{l}\text { Educadores } \\
\text { psicoterapeutas }\end{array}$ & $\begin{array}{l}\text { Herramienta } \\
\text { para evaluar } \\
\text { la pertinencia } \\
\text { de los libros a } \\
\text { usar de acuer- } \\
\text { do con la edad } \\
\text { y problema }\end{array}$ \\
\hline
\end{tabular}




\begin{tabular}{|c|c|c|c|c|c|c|}
\hline $\begin{array}{l}\text { Brewster } \\
\text { (2011) }\end{array}$ & $\begin{array}{l}\text { Tres } \\
\text { bibliotecas } \\
\text { de centros } \\
\text { comu- } \\
\text { nitarios, } \\
\text { Sheffield, } \\
\text { Reino Unido }\end{array}$ & $\begin{array}{l}\text { 4-14 } \\
\text { sesiones } \\
\text { quincenales }\end{array}$ & $\begin{array}{l}\text { Personas con } \\
\text { problemas } \\
\text { de depresión } \\
\text { (adultos) }\end{array}$ & $\begin{array}{l}26 \\
\text { participantes/3 } \\
\text { grupos Grupo 1: } \\
12 \text { personas } \\
\text { Grupo 2: } 8 \\
\text { personas } \\
\text { Grupo 3: } 6 \\
\text { personas }\end{array}$ & $\begin{array}{l}\text { Bibliotecarios, } \\
\text { psicoterapeutas }\end{array}$ & $\begin{array}{l}\text { Cualitativa: } \\
\text { * Observación } \\
\text { participante } \\
\text { `Entrevistas } \\
\text { semiestructu- } \\
\text { radas } \\
{ }^{*} \text { Análisis } \\
\text { descriptivo }\end{array}$ \\
\hline $\begin{array}{l}\text { Deberti Martins } \\
\text { (2013) }\end{array}$ & $\begin{array}{l}\text { Biblioteca } \\
\text { de un centro } \\
\text { de reha- } \\
\text { bilitación, } \\
\text { Montevideo, } \\
\text { Uruguay }\end{array}$ & $\begin{array}{l}\text { Programa } \\
\text { continuo, } \\
\text { portiempo } \\
\text { indefinido }\end{array}$ & $\begin{array}{l}\text { Pacientes en } \\
\text { rehabilitación } \\
\text { por consumo de } \\
\text { drogas (15-35 } \\
\text { años) }\end{array}$ & $\begin{array}{l}3 \text { grupos } \\
8 \text { participantes } \\
\text { c/u }\end{array}$ & $\begin{array}{l}\text { Psicólogos, bibliote- } \\
\text { cólogos y pedadogos }\end{array}$ & $\begin{array}{l}\text { Cualitativa: } \\
\text { observación } \\
\text { participante }\end{array}$ \\
\hline $\begin{array}{l}\text { Mosqueda } \\
\text { Moreno } \\
(2009)\end{array}$ & $\begin{array}{l}\text { Hospital de } \\
\text { Oncología, } \\
\text { CMSXXI, } \\
\text { México, } \\
\text { D.F. }\end{array}$ & $\begin{array}{l}\text { Programa } \\
\text { continuo, } \\
\text { portiempo } \\
\text { indefinido }\end{array}$ & $\begin{array}{l}\text { Pacientes } \\
\text { con cáncer y } \\
\text { sus familiares } \\
\text { (adultos) }\end{array}$ & Indefinido & $\begin{array}{l}\text { Bibliotecólogos, } \\
\text { estudiantes de } \\
\text { servicio social }\end{array}$ & $\begin{array}{l}\text { Cualitativa: } \\
\text { observación } \\
\text { participante }\end{array}$ \\
\hline $\begin{array}{l}\text { Sánchez Trejo } \\
\text { (2009) }\end{array}$ & $\begin{array}{l}\text { Unidad de } \\
\text { rehabilita- } \\
\text { ción física, } \\
\text { IMSS, Méxi- } \\
\text { co, D.F. }\end{array}$ & $\begin{array}{l}\text { Programa } \\
\text { continuo, } \\
\text { por tiempo } \\
\text { indefinido }\end{array}$ & $\begin{array}{l}\text { Pacientes } \\
\text { hospitalizados } \\
\text { (adultos }\end{array}$ & $\begin{array}{l}50 \text { pacientes } \\
5 \text { grupos de } \\
10 \mathrm{c} / \mathrm{u}\end{array}$ & $\begin{array}{l}\text { Bibliotecólogos, } \\
\text { bibliotecarios, } \\
\text { voluntarios }\end{array}$ & $\begin{array}{l}\text { Cualitativa: } \\
\text { encuestas } \\
\text { sobre } \\
\text { preferencias } \\
\text { de lectura }\end{array}$ \\
\hline $\begin{array}{l}\text { Pérez de Rosell } \\
\text { (2011) }\end{array}$ & $\begin{array}{l}\text { Hospital de } \\
\text { Pediatría de } \\
\text { Barqui- } \\
\text { simento, } \\
\text { Venezuela }\end{array}$ & $\begin{array}{l}\text { Programa } \\
\text { continuo, en } \\
\text { periodo de } 6 \\
\text { meses }\end{array}$ & $\begin{array}{l}\text { Niños (1-17 } \\
\text { años) }\end{array}$ & Indefinido & $\begin{array}{l}\text { Médicos, alumnos de } \\
\text { medicina y voluntarios }\end{array}$ & $\begin{array}{l}\text { Cualitativa: } \\
\text { observación } \\
\text { participante y } \\
\text { portafolio de } \\
\text { activodades } \\
\text { Nota: se } \\
\text { evalúa a los } \\
\text { literapeutas, } \\
\text { no a los } \\
\text { pacientes }\end{array}$ \\
\hline $\begin{array}{l}\text { Manecke } \\
\text { (2009) }\end{array}$ & $\begin{array}{l}\text { Biblioteca } \\
\text { central } \\
\text { pública de } \\
\text { Sheffield, } \\
\text { Reino Unido }\end{array}$ & 3 meses & $\begin{array}{l}\text { Usuarios de la } \\
\text { biblioteca } \\
\text { (>16 años) }\end{array}$ & $\begin{array}{l}97 \text { participantes } \\
3 \text { grupos }\end{array}$ & $\begin{array}{l}\text { Bibliotecólogos y } \\
\text { bibliotecarios }\end{array}$ & $\begin{array}{l}\text { Cuantitativa: } \\
\text { cuestiona- } \\
\text { rios sobre } \\
\text { indicadores } \\
\text { de bienestar } \\
\text { y hábitos de } \\
\text { lectura } \\
\text { Cualitativa: } \\
\text { observación } \\
\text { participante }\end{array}$ \\
\hline
\end{tabular}




\begin{tabular}{|c|c|c|c|c|c|c|}
\hline Amer (1999) & $\begin{array}{l}\text { Servicio } \\
\text { endocri- } \\
\text { nología de } \\
\text { un hospital } \\
\text { (ciudad no } \\
\text { especifica- } \\
\text { da), EUA }\end{array}$ & 1 mes & $\begin{array}{l}\text { Niños con DM } \\
\text { y problemas } \\
\text { de crecimiento } \\
\text { (7-16 años) }\end{array}$ & $\begin{array}{l}27 \text { niños de } 2 \\
\text { grupos: } \\
{ }^{*} \text { DM1 } \\
\text { *Problemas de } \\
\text { crecimiento }\end{array}$ & $\begin{array}{l}\text { Doctor en educación, } \\
\text { personal de enfer- } \\
\text { mería }\end{array}$ & $\begin{array}{l}\text { Cualitativa: } \\
\text { entrevista } \\
\text { semiestruc- } \\
\text { turada sobre } \\
\text { reacciones } \\
\text { ante la lectura, } \\
\text { cuestiones de } \\
\text { autoestimay } \\
\text { respuesta ante } \\
\text { burlas }\end{array}$ \\
\hline $\begin{array}{l}\text { Davis } \\
(2005)\end{array}$ & $\begin{array}{l}\text { Bibliotecas } \\
\text { públicas en } \\
\text { Liverpool, } \\
\text { Reino Unido }\end{array}$ & $\begin{array}{l}1 \text { año, } 13 \\
\text { sesiones }\end{array}$ & $\begin{array}{l}\text { Jóvenes de zo- } \\
\text { nas marginales } \\
\text { (16-29 años) }\end{array}$ & $\begin{array}{l}20 \text { grupos } \\
\text { pequeños (no } \\
\text { se especifica } \\
\text { número) }\end{array}$ & No especificado & $\begin{array}{l}\text { Cuantitativa: } \\
\text { número de } \\
\text { libros leídos } \\
\text { y número de } \\
\text { personas en- } \\
\text { tusiasmadas } \\
\text { Cualitativa: } \\
\text { entrevistas } \\
\text { semiestructu- } \\
\text { radas }\end{array}$ \\
\hline $\begin{array}{l}\text { Sharma et al. } \\
\text { (2014) }\end{array}$ & $\begin{array}{l}\text { Clínica } \\
\text { Mayo, } \\
\text { Rochester } \\
\text { Minnesota, } \\
\text { EUA }\end{array}$ & $\begin{array}{l}12 \\
\text { semanas }\end{array}$ & $\begin{array}{l}\text { Empleados de } \\
\text { un hospital }\end{array}$ & 37 adultos & No especificado & $\begin{array}{l}\text { Cuantitativa: } \\
\text { instrumentos } \\
\text { estanda- } \\
\text { rizados: } \\
\text { Connor-Da- } \\
\text { vidson Resi- } \\
\text { lience Scale; } \\
\text { Perceived } \\
\text { Stress Scale; } \\
\text { Smith Anxiety } \\
\text { Scale; Linear } \\
\text { Analogue } \\
\text { Self-Assess- } \\
\text { ment Scale; } \\
\text { and Mindful } \\
\text { Attention } \\
\text { Awareness } \\
\text { Scale }\end{array}$ \\
\hline $\begin{array}{l}\text { Reeves } \\
(2010)\end{array}$ & $\begin{array}{l}\text { Dos clínicas } \\
\text { familiares } \\
\text { (ciudades } \\
\text { no especi- } \\
\text { ficadas), } \\
\text { Reino Unido }\end{array}$ & 7 semanas & $\begin{array}{l}\text { Pacientes } \\
\text { adultos }\end{array}$ & $\begin{array}{l}43 \text { participantes, } \\
2 \text { grupos } \\
{ }^{*} \text { en tratamiento } \\
{ }^{*} \text { control }\end{array}$ & Psicólogos & $\begin{array}{l}\text { Cuantitativa: } \\
\text { cuestionarios } \\
\text { analizados con } \\
\text { SPSS } \\
\text { Cualitativa: } \\
\text { entrevista } \\
\text { semiestructu- } \\
\text { rada. } \\
3 \text { momentos } \\
\text { de evaluación: } \\
\text { al inicio, al } \\
\text { término de la } \\
\text { intervención } \\
\text { y } 3 \text { meses } \\
\text { después }\end{array}$ \\
\hline
\end{tabular}


Como puede verse en la Tabla 2, las prácticas y formas de evaluar los talleres de biblioterapia son muy variadas. Hay quienes la llevan a cabo en contextos hospitalarios, mientras que otros la practican en bibliotecas públicas, centros comunitarios o de rehabilitación. De entre los que la utilizan en ámbitos clínicos, algunos lo hacen con pacientes con una aflicción en común, otros con pacientes hospitalizados, algunos más con pacientes ambulatorios y los familiares que los acompañan. También existen casos en que la biblioterapia hospitalaria se imparte al personal que allí labora, como un mecanismo para reducir los niveles de estrés propios de su profesión y del ambiente institucional. En aquél y otros contextos a veces se trabaja con adultos, a veces con niños y en ocasiones con grupos mixtos en una franja etaria amplia. Asimismo, la biblioterapia se ofrece al público en general en bibliotecas públicas, con el afán de mejorar su vida cotidiana. En muchos de estos casos, los biblioterapeutas son voluntarios y los gobiernos nacionales o locales (o incluso miembros de la misma comunidad) aportan recursos económicos para adquirir los acervos bibliográficos y negociar espacios adecuados para la terapia de lectura. Cabe mencionar que las maneras de evaluar los efectos de la biblioterapia no siguen un patrón estandarizado. Hay investigadores que apuestan por una visión positivista, que aspira a arrojar datos duros sobre los beneficios de la terapia con libros por medio de indicadores estandarizados de ansiedad, estrés y bienestar. Otros, de manera deliberada o simplemente por omisión, no reportan evaluación alguna de sus programas de biblioterapia, aunque sí hablan de sus efectos favorables -a veces inespecíficos. Finalmente existen investigaciones que pueden clasificarse en un tercer grupo: aquellas que combinan valoraciones cuantitativas (cuestionarios, conteo de número de libros o número de lectores) con estimaciones cualitativas (observaciones, entrevistas, anécdotas) sobre las consecuencias de la biblioterapia, no sólo para quienes la reciben, sino también para quienes la imparten.

Un punto de coincidencia importante entre todas las publicaciones analizadas para este artículo es que la biblioterapia tiene efectos positivos, tanto en quienes la reciben como en quienes la imparten. Pérez de Rosell (2011: 10), por ejemplo, reporta que los biblioterapeutas (en ese caso estudiantes de medicina) lograron estrechar sus vínculos de empatía con los niños del hospital. De forma similar, Amer (1999: 93) describe su propia sensación de bienestar al notar las reacciones positivas de sus dos poblaciones de niños. Es importante destacar que en las fuentes consultadas, en especial aquellas provenientes de países de América Latina, predominan los informes de tipo anecdótico y se reportan pocas evaluaciones integrales sobre los efectos de la biblioterapia. Esto podría explicar en parte la aparente renuencia a implementarla de manera sistemática en nuestro país, así como la falta de 
organismos que patrocinen este tipo de programas por periodos más largos. Por otra parte, pudimos observar que son muy pocas las investigaciones que dan seguimiento a largo plazo a un mismo grupo de estudio, para saber qué tan duraderos son los cambios positivos que se observan. Haciendo una analogía con otras formas de terapia, resulta lógico pensar que para lograr cambios duraderos se requiere de constancia y de intervenciones periódicas de refuerzo. Es aquí donde parecería existir una suerte de círculo vicioso frenando la creación de más programas de biblioterapia: al no existir financiamiento suficiente para organizar y seguir en el tiempo talleres de terapia de lectura, resulta imposible probar de manera tangible sus beneficios, lo cual a su vez dificulta la labor de impulsar más proyectos de este tipo, con financiamiento gubernamental y privado. Una posible solución para comenzar a romper el círculo en nuestro país podría ser la implementación de programas institucionales de servicio social bien coordinados, en donde una generación releve a la siguiente de manera que pueda darse continuidad a los talleres y pudieran notarse sus beneficios así como sus debilidades.

\section{Proyecciones y PROSPECTIVAS}

A lo largo de este artículo hemos explorado los diversos conceptos, facetas y tendencias de la biblioterapia en México y el mundo, reportados en el ámbito académico. Nuestra revisión sistemática de informes, artículos de investigación y ensayos de divulgación nos lleva a concluir que la biblioterapia es una intervención de bajo costo y amplio espectro, con gran potencial para su aplicación extensiva en distintos espacios y con audiencias muy diversas. Si bien el sufijo "terapia" pareciera restringirla como técnica de tratamiento para padecimientos bien identificados, como se ha visto la biblioterapia es también un método complementario en procedimientos terapéuticos, así como una práctica que puede beneficiar a individuos sin enfermedad aparente. Un elemento en común entre la mayoría de los programas que se describen en la bibliografía consultada es la convicción de que cuando la lectura se vuelve un hábito cotidiano tiene repercusiones profundas en la vida de las personas, y más aún cuando se realiza como actividad compartida. Este último punto es, a nuestro parecer, una de las grandes fortalezas de la biblioterapia, en especial cuando la discusión grupal se combina con la reflexión solitaria que caracteriza a la lectura individual. Quizá la cualidad fundamental de este tipo de terapia sea precisamente su peculiar combinación de introspección colectiva, la cual permite activar zonas cognitivas y emocionales del cerebro como ninguna otra actividad humana en la primera etapa, así como compartir puntos de vista y ampliar las primeras impresiones por medio de la discusión con otros. 
El alcance y la trascendencia potencial de la biblioterapia apenas comienzan a vislumbrarse. Por lo tanto, resulta de interés efectuar más intervenciones con diferentes poblaciones y realizar más investigaciones a fondo para probar su efectividad, en especial a mediano y largo plazo, en nuestro país. Dado que la lectura es una actividad relativamente sencilla y los materiales para llevarla a cabo son de una variedad casi infinita, la biblioterapia resulta un modelo muy atractivo para probarse y afinarse en diferentes contextos, con la posibilidad de beneficiar a públicos muy heterogéneos. Asimismo, resultaría muy interesante y provechoso explorar la posibilidad de aprender de y complementar la riqueza de la experiencia biblioterapéutica con prácticas como la arteterapia, danzaterapia y prácticas similares. Como lo habíamos mencionado en la sección previa, entre las posibilidades que se antojan factibles para comenzar a fortalecer y en un futuro arraigar proyectos duraderos de biblioterapia en México están los programas de servicio social obligatorio en las universidades públicas a lo largo del país. Implementar talleres biblioterapéuticos de este tipo podría garantizar no sólo la continuidad a mediano y largo plazos, sino también favorecer la interdisciplinaridad al permitir el reclutamiento y entrenamiento de facilitadores de talleres con diversas formaciones académicas. En última instancia, un primer paso crucial es comenzar a conocer y divulgar el sustento teórico, metodologías y mejores experiencias de la biblioterapia, para generar cada vez más interés en llevarla a la práctica. Con este artículo esperamos haber contribuido en este sentido.

\section{REFERENCIAS}

Akinola, Ajayi Nathaniel. 2014. "Bibliotherapy as an Alternative Approach to Children's Emotional Disorders”. Creative Education, 1281-1285.

Amer, Kim. 1999. "Bibliotherapy: Using Fiction to Help Children in Two Populations Discuss Feelings". Pediatric Nursing 25: 91-95.

Angelotti, Michael. 1985. "Uses of Poetry and Adolescent Literature in Therapy for Adolescents", en Adolescents, Literature, and Work With Youth, Pamela Weiner y Ruth Stein (eds.), 27-38. Londres: Routledge.

Anglin, Carlita. 2008. "Providing Pediatric Psychosocial Support through Patient Library Services in an Outpatient Hematology/Oncology Clinic”. Primary Psychiatry 15: 78-83.

Apodaca, Timothy R. y William R. Miller. 2003. "A Meta-analysis of the Effectiveness of Bibliotherapy for Alcohol Problems". Journal of Clinical Psychology 59 (3): 289-304.

Aristóteles. S. f./1948. Arte poética. Trad. De José Goya y Muniain. Buenos Aires: TRADIA-BUAP.

Beatty, William. 1962. "A Historical Review of Bibliotherapy". Library Trends 11: 106-117. 
Bernardino, M. R. 2012. "Biblioterapia com Crianças com Câncer”. Informação E Informação 17 (3): 198-210.

Bernstein, J. P. 1989. "How books can help young children cope”. En Children's literature: Resource for the Classroom, M. K. Rudman (ed.), 159-173. Norwood, MA: Christopher and Gordon Publishers.

Betzalel, Nurit y Zipora Schechtman. 2010. "Bibliotherapy Treatment for Children with Adjustment Difficulties: A Comparison of Affective and Cognitive Bibliotherapy" Journal of Creativity in Mental Health 5 (4): 426-439.

Bodart, Joni R. 2006. "Books That Help, Books That Heal: Dealing With Controversy in YA Literature". Young Adult Library Services 5 (1): 31-34.

Bonnycastle, Stephen. 2001. "Bibliotherapy in Action: A Reader's Developing Responses to Two Stories About Obsessional Love”. Textual Studies in Canada 13/14: 1-13.

Brewster, Elizabeth. 2011. "An Investigation of Experiences of reading for Mental Health and Well-Being and Their Relation to Models of Bibliotherapy". Tesis, The University of Sheffield.

Brewster, Elizabeth. 2009. "Books on Prescription: Bibliotherapy in the United Kingdom”. Journal of Hospital Librarianship 9: 399-407.

Chávez, Carlos. 2008. "La publicidad catapulta a Gandhi”. CNN Expansión, 31 de mayo de 2008. http://www.cnnexpansion.com/negocios/2008/05/31/el-exito-amarillo-de-gandhi

Chester E. Tillman. 1984. "Bibliotheraphy for Adolescents: An Annotated Research Review”. Journal of Reading 27: 713-719.

Cohen, L. J. 1987. "Bibliotherapy. Using Literature to Help Children Deal with Difficult Problems". Journal of Psychosocial Nursing in Mental Health Services 25: 20-24.

Cohen, L. J. 1994. "Bibliotherapy: a Valid Treatment Modality. Research-Based Practice". Journal of Psychosocial Nursing in Mental Health Services 32: 40-44.

Conaculta. 2006. Encuesta Nacional de Lectura 2006. México: Consejo Nacional para la Cultura y las Artes.

Conaculta. 2012. Encuesta Nacional de Lectura 2012. México: Consejo Nacional para la Cultura y las Artes.

Crothers, Samuel McChord. 1916. "A Literary Critic", Atlantic Monthly 118: 291-301.

Cujipers, Pieter. 1997. "Bibliotherapy in Unipolar Depression: A Meta-analysis". Journal of Behaviour Therapy and Experimental Psychiatry 28 (2): 139-147.

Davis, Jane. 2005. Step Into Books: Final Report Into the Get Into Reading Project 2004-5. Liverpool.

Diodoro Sículo. 2001. Biblioteca bistórica, vol. I. Trad. y notas de Francisco Parreu Alasá. Madrid: Editorial_Gredos.

Deberti Martins, Cristina. 2011. "Leer: un derecho... también en el hospital”. Información, Cultura y Sociedad 25: 145-152.

Deberti Martins, Cristina. 2013. "Los Libros Muerden: Biblioterapia En El Portal Amarillo". Informatio 18: 21-30.

Dobson, R. 2003. “GPs Prescribe Self-help Books for Mental Health Problems”. BMJ 326: 1285.

Duffy, John, Jo Haslam, Lesley Holl y Julie Walker. 2013. Well into Words: Bibliotherapy toolkit. Kirklees, Huddersfield: Kirklees Library.

Dysart-Gale, D. 2008. "Lost in Translation: Bibliotherapy and Evidence-Based Medicine”. Journal of Medical Humanities 29: 33-43. 
Favazza, A. R. 1996. "Bibliotherapy: a Critique of the Literature”. Bulletin of the Medical Library Association 54: 138-141.

Floyd, Mark. 2003. "Bibliotherapy as an Adjunct to Psychotherapy for Depression in Older Adults". JCLP/In Session: Psychotherapy in Practice 59 (2): 187-95.

Fluck, Winifried. 2003. "Fiction and Justice”. New Literary History 34.1: 19-42.

Fulford, K. W. 2011. "Bringing together values-based and evidence-based medicine: UK Department of Health Initiatives in the 'Personalization' of Care". Journal of Evaluation of Clinical Practice 17 (2): 341-343.

García, Betty y Marilyn Bregoli. 2000. "The Use of Literary Sources in the Preparation of Clinicians for Multicultural Practice". Journal of Teaching in Social Work 20: 77-102.

Gavigan, Karen. 2012. "Caring Through Comics: Graphic Novels and Bibliotherapy for Grades 6-12”. Knowledge Quest 40: 78-80.

Gladding, Samuel. 2005. Counselling as an Art, 4a. ed. Alexandria, VA: American Counseling Association.

Gold, Joseph. 1990. Read for Your Life: Literature as a Life Support System. Markham: Fitzhenry and Whiteside.

Gregory, Robert J., S. Schwer-Canning, T. W. Lee y J. C. Wise. 2004. "Cognitive Bibliotherapy for Depression: a Meta-analysis”. Professional Psychology: Research and Practice 35 (3): 275-80.

Gubert, Betty K. 1993. "Sadie Peterson Delaney: Pioneer Bibliotherapist”. American Libraries 24: 124.

Hicks, Debbie. 2006. "An Audit of Bibliotherapy/Books on Prescription Activity in England”. Londres: Arts Council England and The Museums Libraries and Archives Council, 2006, 1-129.

Inegi. 2014. Estadísticas a propósito del día mundial del libro y el derecho de autor, 21 de abril de 2014. http://www.inegi.org.mx/inegi/contenidos/espanol/prensa/ Contenidos/ estadisticas/2014/libro0.pdf

Iser, Wolfgang. 1989. Prospecting from Reader Response to Literary Anthropology. Baltimore: The Johns Hopkins University Press.

Jalongo, Mary Renk. 1983. "Bibliotherapy: Literature to Promote Socioemotional Growth". The Reading Teacher 36: 796-803.

Jones, F. A. 2002. "The Role of Bibliotherapy in Health Anxiety: an Experimental Study”. British Journal of Community Nursing 7: 498-504.

Jones, Jami L. 2006. "A Closer Look at Bibliotherapy”. Young Adult Library Services 5 (1): 24-27.

Kaywell, Joan F. 2004. Using Literature to Help Troubled Teenagers Cope with Abuse Issues. Westport, Connecticut: Greenwood Press.

Kramer, Karin. 2009. "Using Self-Help Bibliotherapy in Counselling”. Tesis, University of Lethbridge.

Levin, L. y R. Gildea. 2013. "Bibliotherapy: Tracing the Roots of a Moral Therapy Movement in the United States From the Early Nineteenth Century to the Present”. Journal of Medical Library Association 101: 89-91.

Louie, Belinda Y. y Douglas H. Louie. 1992. "Empowerment through Young-Adult Literature". The English Journal 81 (4): 53-56.

Lu, Ya. 2008. "Helping Children Cope: What Is Bibliotherapy?" Children E Libraries: The Journal of the Association for Library Service to Children 6: 47-49. 
Manecke, Ute. 2009. “Bibliotherapy: The Restorative Power of Reading”. Tesis, The University of Sheffield.

Marrs, R. W. 1995. "A Meta-Analysis of Bibliotherapy Studies”. American Journal of Community Psychology 23: 843-870.

McCann, Terence V. y Dan I. Lubman. 2014. "Qualitative Process Evaluation of a Problem-Solving Guided Self-Help Manual for Family Carers of Young People with First-Episode Psychosis". BMC Psychiatry 14: 14-168.

McChord Crothers, Samuel. 1916. "A Literary Critic”. Atlantic Monthly 118: 291-301.

McMillen, P. S. 2006. "A Therapeutic Collaboration: The Bibliotherapy Education Project at Oregon University”. OLA Quarterly 12: 14-15.

Mosqueda Moreno, María Alicia. 2009. "La biblioterapia en pacientes con cáncer y tratamiento de radioterapia”. Informe académico, Licenciatura en Bibliotecología. México: Universidad Nacional Autónoma de México.

OMS (Organización Mundial de la Salud). 1948. "Preámbulo a la Constitución de la Organización Mundial de la Salud, adoptada por la Conferencia Internacional de Salud, Nueva York, 19-22 de junio, 1946, puesta en vigor el 7 de abril de 1948”, Registros oficiales de la Organización Mundial de la Salud 2: 100.

Palladino, Dianne K. y Vicki S. Helgeson. 2012. "Friends or Foes? A Review of Peer Influence on Self-Care and Glycemic Control in Adolescents With Type 1 Diabetes". Journal of Pediatric Psychology 37 (5): 591-603.

Pardeck, J. T. 1990. "Using Bibliotherapy in Clinical Practice with Children”. Psychological Reports 67: 1043-1049. PM: 2287656.

Pehrsson, Dale-Elizabeth. 2005. "Fictive Bibliotherapy and Therapeutic Storytelling with Children Who Hurt", en Creative Interventions in Grief and Loss Therapy: When the Music Stops, a Dream Dies, Thelma Duffey (ed.), 273-286. Philadelphia: Haworth Press.

Pérez de Rosell, Milagros. 2011. "Literapia Como Práctica Terapéutica De Los Estudiantes De Autodesarrollo Del DCS En El Hospital Universitario De Pediatría 'Agustín Zubillaga' De Barquismeto. Una Experiencia Educativa”. Boletín Médico de Postgrado 28: 109-117.

Reeves, T. 2010. "A Controlled Study of Assisted Bibliotherapy: an Assisted SelfHelp Treatment for Mild to Moderate Stress and Anxiety". Journal of Psychiatric and Mental Health Nursing 17: 184-190.

Reitz, Joan M. 2014. Dictionary for Library and Information Science. Sta. Bárbara, CA: ABC Clio.

Rodríguez-Martin, B. C., Ainsleidy Gómez-Quintana, Glendy Díaz-Martínez y Osana Molerio-Pérez. 2013. "Bibliotherapy and Food Cravings Control". Appetite 65: 90-95.

Rozalski, Michael, Angela Stewart y Jason Miller. 2010. "Bibliotherapy: Helping Children Cope With Life’s Challenges”. Kappa Delta Pi Record 47: 33-37.

Rushdie, Salma. 1990. In Good Faith. Londres: Granta.

Russell, David H. y Caroline Shrodes. 1950. The School Review 58 (6): 335-342.

Salaverti Pitarch, Pepa. 1999. "La Biblioteca De Usuarios Del Hospital Universitario La Fe De Valencia”. Métodos de Información 6: 74-76.

Sánchez Trejo, Margarita. 2009. "Propuesta de un programa de biblioterapia para pacientes hospitalizados en la Unidad de Medicina Física y Rehabilitación Región Centro del Instituto Mexicano del Seguro Social”. Tesis, Universidad Nacional Autónoma de México. 
Schechtman, Zipora. 2009. Treating Child and Adolescent Aggression Through Bibliotherapy. New York: Springer.

Seitz, E. 2008. "A biblioterapia na humanização da assistência hospitalar do Hospital Universitário da Universidad Federal de Santa Catarina”. ETD Educação Temática Digital 9 (2): 145-169.

Sharma, Varun, Amit Sood, Kavita Prasad, Laura Loehrer, Darrell Schroede y Bauer Brent. 2014. "Bibliotherapy to Decrease Stress and Anxiety and Increase Resilience and Mindfulness: a Pilot Trial". Explore (NY) 10: 248-252.

Shrodes, Caroline. 1950. "Bibliotherapy: A Theoretical and Clinical Experimental Study”. Diss., University of California.

Shrodes, Caroline. 2005. "Bibliotherapy", Reading Teacher 9: 24-29.

Silverberg, L. I. 2003. "Bibliotherapy: the Therapeutic Use of Didactic and Literary Texts in Treatment, Diagnosis, Prevention, and Training”. Journal of the American Ostheopathic Association 103: 131-135.

Thirion, Vivianne. 2011. Biblioterapia: La Lectura Cura. México: SEP.

Thomas, S. P., ed. 2011. "Bibliotherapy: New Evidence of Effectiveness". Issues in Mental Health Nursing 32: 191.

Tukhareli, Natalia. 2016. Read to Connect. Portal de información en línea. http://readtoconnect.ca

Turner, Jane. 2008. "Bibliotherapy for Health and Wellbeing: an Effective Investment”. Aplis 21:56-61.

Villamil, Jenaro. 2013. "Entre 108 países, México es penúltimo lugar en lectura”. Revista Proceso, 23 de abril de 2013. http://www.proceso.com.mx/ ?p=339874.

Weimer, Elizabeth. 2010. "The Power of Books in a Children's Hospital". Children and Libraries, 20-21.

Para citar este texto:

Castro Santana, Anaclara y Nelly Altamirano Bustamante. 2018. “¿Leer para estar bien?: prácticas actuales y perspectivas sobre la biblioterapia como estrategia educativo-terapéutica”. Investigación Bibliotecológica: archivonomía, bibliotecología e información 32 (74): 171-192.

http://dx.doi.org/10.22 201/iibi.24488321xe.2018.74.57918 